\title{
Ultrasonic 3-D Vector Flow Method for Quantitative In Vivo Peak Velocity and Flow Rate Estimation
}

Holbek, Simon; Ewertsen, Caroline; Bouzari, Hamed; Pihl, Michael Johannes; Hansen, Kristoffer Lindskov; Stuart, Matthias Bo; Thomsen, Carsten; Nielsen, Michael Bachmann; Jensen, Jørgen Arendt

Published in:

IEEE Transactions on Ultrasonics, Ferroelectrics and Frequency Control

Link to article, DOI:

10.1109/TUFFC.2016.2639318

Publication date:

2017

Document Version

Peer reviewed version

Link back to DTU Orbit

Citation (APA):

Holbek, S., Ewertsen, C., Bouzari, H., Pihl, M. J., Hansen, K. L., Stuart, M. B., Thomsen, C., Nielsen, M. B., \& Jensen, J. A. (2017). Ultrasonic 3-D Vector Flow Method for Quantitative In Vivo Peak Velocity and Flow Rate Estimation. IEEE Transactions on Ultrasonics, Ferroelectrics and Frequency Control, 64(3), 544-554.

https://doi.org/10.1109/TUFFC.2016.2639318

\section{General rights}

Copyright and moral rights for the publications made accessible in the public portal are retained by the authors and/or other copyright owners and it is a condition of accessing publications that users recognise and abide by the legal requirements associated with these rights.

- Users may download and print one copy of any publication from the public portal for the purpose of private study or research.

- You may not further distribute the material or use it for any profit-making activity or commercial gain

- You may freely distribute the URL identifying the publication in the public portal 


\title{
Ultrasonic 3-D vector flow method for quantitative in vivo peak velocity and flow rate estimation
}

\author{
Simon Holbek, Caroline Ewertsen, Hamed Bouzari, Michael Johannes Pihl, Kristoffer Lindskov Hansen, \\ Matthias Bo Stuart, Carsten Thomsen, Michael Bachmann Nielsen, and Jørgen Arendt Jensen
}

\begin{abstract}
Current clinical ultrasound systems are limited to show blood flow movement in either 1-D or 2-D. In this paper, a method for estimating 3-D vector velocities in a plane using the Transverse Oscillation (TO) method, a $32 \times 32$ element matrix array, and the experimental ultrasound scanner SARUS is presented. The aim of this paper is to estimate precise flow rates and peak velocities derived from 3-D vector flow estimates. The emission sequence provides 3-D vector flow estimates at up to 1.145 frames per second in a plane, and was used to estimate 3-D vector flow in a cross sectional image plane. The method is validated in two phantom studies, where flow rates are measured in a flow-rig, providing a constant parabolic flow, and in a straight-vessel phantom $(\oslash=8 \mathrm{~mm})$ connected to a flow pump capable of generating time varying waveforms. Flow rates are estimated to be $82.1 \pm 2.8 \mathrm{~L} / \mathrm{min}$ in the flow-rig compared with the expected $79.8 \mathrm{~L} / \mathrm{min}$, and to $2.68 \pm 0.04 \mathrm{~mL} /$ stroke in the pulsating environment compared with the expected $2.57 \pm 0.08$ $\mathrm{mL} / \mathrm{stroke}$. Flow rates estimated in the common carotid artery of a healthy volunteer are compared with MRI measured flow rates using a 1-D through-plane velocity sequence. Mean flow rates were $333 \pm 31 \mathrm{~mL} / \mathrm{min}$ for the presented method and 346 $\pm 2 \mathrm{~mL} / \mathrm{min}$ for the MRI measurements.
\end{abstract}

\section{INTRODUCTION}

C ARDIOVASCULAR diseases account for $30 \%$ of global deaths [1]. A better understanding of the true blood flow dynamics could increase the chances of diagnosing critical diseases at an earlier stage, thereby improving treatment success rates. Since vascular flow can propagate in all three dimensions, 3-D vector flow imaging at a very high frame rate is a necessity for providing operators with the complete velocity field in time and space.

Currently, velocity estimation in most commercial scanners is limited to estimating only the axial velocity using, e.g., spectral Doppler, which requires manual angle correction by the operator to obtain the true velocity. Although the angle correction may introduce critical errors [2], [3], spectral Doppler is a widely used tool for peak velocity and flow rate estimation. However, angle correction schemes are not required for 3-D vector flow imaging (VFI) techniques.

Various methods for estimating 2-D vector flow or 2-D flow dynamics have been proposed; speckle tracking [4], synthetic

This work was financially supported by grants 82-2012-4 from the Danish National Advanced Technology Foundation and from BK Ultrasound ApS (Herlev, Denmark).

S. Holbek, Hamed, Bouzari, M.J. Pihl, M. B. Stuart and J.A. Jensen are with the Center for Fast Ultrasound Imaging, Department of Electrical Engineering, Technical University of Denmark, Kgs. Lyngby, Denmark.

C. Ewertsen, K.L. Hansen, C. Thomsen and M.B. Nielsen are with Department of Radiology, Copenhagen University Hospital, Rigshospitalet, Copenhagen E, Denmark aperture flow [5], [6], [7], plane waves [8], [9], [10], Doppler vortography [11], multi beam Doppler [12], and Transverse Oscillation (TO) [13], [14]. 2-D vector flow gives a more realistic estimation of the actual flow, but does not provide information about the out-of-plane velocity component.

Some of the methods proposed for vector flow estimation can also be extended to 3-D, but all of them require data sampled in two dimensions to estimate all three velocity components. Several 2-D sampling techniques have been proposed, for instance triple-beam lens transducers [15], row-column addressed 2-D arrays [16] [17], and a 2-D piezoelectric matrix array [18].

A 2-D piezoelectric transducer was used in our previous work, which showed that all three velocity components can be obtained for two crossing planes [19] using 3-D implementation of the TO method [20] [21], and Provost et al. estimated the axial velocities in a full volume using Doppler techniques with a similar transducer [22]. The true 3-D velocity vector provides the physician with valuable information about the complex flow, without relying on transducer orientation.

Currently, state of the art magnetic resonance imaging (MRI) can provide 4-D blood flow velocity estimates for large volumes [23], and it is often referred to as the gold standard for accurate non-invasive blood velocity estimation. Several studies have compared blood flow estimates from an ultrasound (US) scanning with similar observations obtained from MRI [24], [25]. They showed that US estimates are comparable to MRI estimates, although with a positive bias. One of the drawbacks of MRI is the long acquisition time, where data are averaged over 10-15 min [23][26]. This makes it less suitable for estimation of peak velocities, and also, the cost of the scan is significantly higher than for a US scan.

In this work, the precision of 3-D US vector flow estimation is validated for pulsating flow. First in two phantom measurements, and second, when compared against MRI by measuring the flow rates in the common carotid artery in a healthy volunteer.

Previous work with the 2-D matrix probe by our group has covered a parametric study of an M-mode sequence using the 3-D TO method for both a simulation setup, and in an experimental setup, where the blood flow estimation performance of laminar parabolic flow was investigated. [20], [21]. This paper is based on conference proceedings [27] and expands on previous work by introducing an emissions sequence with twice the amount of flow lines, twice the acquisition time, and provides B-mode volumes obtained from synthetic aperture techniques. The improvements were achieved primarily by 
adjusting the phase shift estimator to lie within the expected velocity range and by sampling data from a shorter range than previously. The revised approach was obtained without sacrificing frame rate or resolution. Validation of the derived angle independent 3-D vector flow estimates are compared with a gold standard MRI through-plane measurement.

\section{3-D VFI METHODS}

This Section introduces the methods used for the experimental US measurements, in terms of emission sequence, data processing and theoretical properties of the TO velocity estimator. A summary of the variables used is seen in Table I. The study was performed after approval by the Danish National Committee on Biomedical Research Ethics (H-12014-FSP-072).

\section{A. Emission sequence}

Considering a conventional transmit sequence composed of the unique flow lines $F_{i}, i=1,2, \ldots N$, and the unique Bmode emissions $B_{j}, j=1,2, \ldots M$ (see Fig. 1), a schematic representation of one full transmit cycle is written as

$$
\begin{gathered}
\overbrace{F_{1} \rightarrow F_{1} \cdots \rightarrow F_{1}}^{\text {Ensemble length, } N_{e}} \\
F_{2} \rightarrow F_{2} \cdots \rightarrow F_{2} \\
\vdots \quad \vdots \quad \vdots \\
F_{N} \rightarrow F_{N} \cdots \rightarrow F_{N} \\
B_{1} \rightarrow B_{2} \rightarrow B_{3} \rightarrow B_{4} \cdots \rightarrow B_{M},
\end{gathered}
$$

where the ensemble length $N_{e}$, is the number of emissions used for each estimate (Flow or B-mode). Each unique flow line $F_{i}$ is emitted sequentially a pre-defined amount of times until the next round of a different flow line is emitted. This continues until all $F_{N}$ transmit events are completed. The flow transmissions are followed by emitting the $M$ B-mode emissions. After each cycle of a unique flow emissions e.g. $F_{1}$, velocity estimates along this direction can be obtained. The same argument holds for the remaining $N$ flow lines. After the $M$ B-mode emissions, one B-mode image or volume can be obtained. Finally, the velocity information along all the estimated directions are assembled and can be combined with the B-mode image. Such a sequence has a high detectable velocity range, but at the cost of a low frame rate, since estimates are made line-by-line.

An alternative emission sequence exists, where all identical transmit events are spaced equally in time. This type of sequence is considered a continuous data scheme [5], [28]. Continuous data can be obtained using synthetic aperture imaging, plane waves, focused emissions, as long as the transmit sequence is repeated periodically.

One of the advantages of continuous data are that very high frame rates in the $\mathrm{kHz}$ range may be obtained [5], [7], [14], [28], [29]. The high frame rate is obtained by a sliding window that can be moved along the processed data in such a way

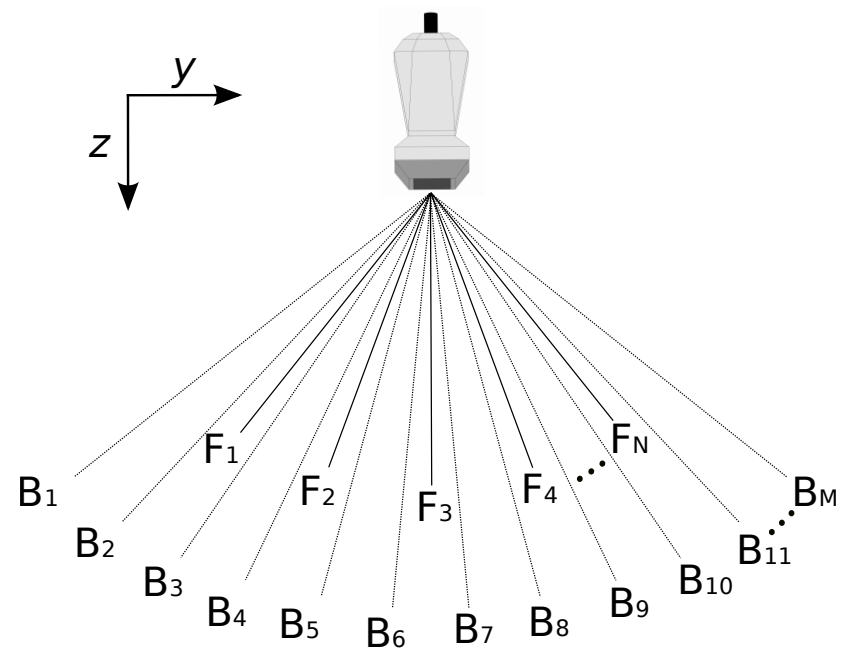

Fig. 1: Illustration of a transmit sequence containing both flow emissions $(F)$ and B-mode emissions $(B)$. Depending on the design of the sequence it is possible to acquire continuous data.

that whenever a new acquisition has been made, it replaces the oldest sample in the velocity estimator [30], and several adaptive algorithms may be applied to improve the estimates. For instance, when continuous data are available, the number of emissions needed in the estimator may vary over time or more advanced echo canceling filters may be used.

The idea is that the duration between emissions of two identical flow lines $F_{i}$, or two identical B-mode emissions $B_{j}$, should be the same at all times. This is obtained by emitting the flow lines $F_{1 \rightarrow N}$ consecutively, followed by emitting the B-mode emission $B_{1}$. Next, the flow lines $F_{1 \rightarrow N}$ are emitted, followed by the B-mode emission $B_{2}$ etc. When the last unique B-mode emission $B_{M}$ has been transmitted, the sequence repeats itself from the beginning. A schematic representation of the emission sequence would be:

$$
\begin{array}{r}
F_{1} \rightarrow F_{2} \rightarrow F_{3} \rightarrow F_{4} \cdots \rightarrow F_{N} \rightarrow B_{1} \rightarrow \\
F_{1} \rightarrow F_{2} \rightarrow F_{3} \rightarrow F_{4} \cdots \rightarrow F_{N} \rightarrow B_{2} \rightarrow \\
\vdots \quad \vdots \quad \vdots \quad \vdots \quad \vdots \quad \\
\vdots \quad \vdots \\
F_{1} \rightarrow F_{2} \rightarrow F_{3} \rightarrow F_{4} \cdots \rightarrow F_{N} \rightarrow B_{M} \rightarrow
\end{array}
$$

The presented emission sequence fulfills the requirement of having the same time span between two similar transmit events, and can acquire continuous data. Based on the above mentioned principles an interleaved emission sequence with $\mathrm{M}=36$ and $N=10$ was designed. The flow lines were steered from $-15^{\circ}$ to $15^{\circ}$ in steps of $3.3^{\circ}$. Electronic delay profiles were applied on all 1024 elements in transmit to generate a focal point at $35 \mathrm{~mm}$ depth for the flow sequence. The 36 B-mode emissions with virtual point sources placed $15 \mathrm{~mm}$ behind the transducer at different locations were applied to generate a $60^{\circ} \times 60^{\circ}$ field-of-view volume using synthetic aperture imaging techniques, Fig. 2. From each B- 
TABLE I: Transducer and Emissions Sequence Setup

\begin{tabular}{|c|c|c|c|c|c|c|}
\hline \multicolumn{2}{|c|}{ Transducer } & \multicolumn{3}{|c|}{ Flow Parameters } & \multicolumn{2}{|c|}{ B-mode } \\
\hline Parameter & Value & Parameter & Phantom & In vivo & Parameter & Value \\
\hline Transucer type & 2-D phased array & Excitation signal & 8 cycled & sin. Hanning weighted & Excitation signal & 2 cycled $\sin$ \\
\hline No. of elements in $\mathrm{x}$ & 35 ( 3 inactive rows) & Frequency & & $3 \mathrm{MHz}$ & Frequency & $3 \mathrm{MHz}$ \\
\hline No. of elements in $y$ & 32 & Flow lines $N$ & & 10 & B-mode emissions $M$ & $6 \times 6$ \\
\hline Pitch in $\mathrm{x} \& \mathrm{y}$ & $0.3 \mathrm{~mm}$ & $f_{p r f}$ & $5 \mathrm{kHz}$ & $12.6 \mathrm{kHz}$ & Virtual source & $-1.5 \mathrm{~cm}$ \\
\hline Height & $0.278 \mathrm{~mm}$ & Steering angles & & {$\left[-15^{\circ}: 15^{\circ}\right]$} & Field-of-view & $60^{\circ} \times 60^{\circ}$ \\
\hline Kerf & $0.022 \mathrm{~mm}$ & Emissions per estimate & & 16 & Emissions per volume & 36 \\
\hline Sampling frequency & $17.5 \mathrm{MHz}$ & $\lambda_{x} \& \lambda_{y}$ & 3.2 & $1 \mathrm{~mm} \& 2.55 \mathrm{~mm}$ & Tx apodization & Hann \\
\hline Center frequency & $3.5 \mathrm{MHz}$ & Focal depth & & $35 \mathrm{~mm}$ & Rx apodization & Hann. \\
\hline
\end{tabular}

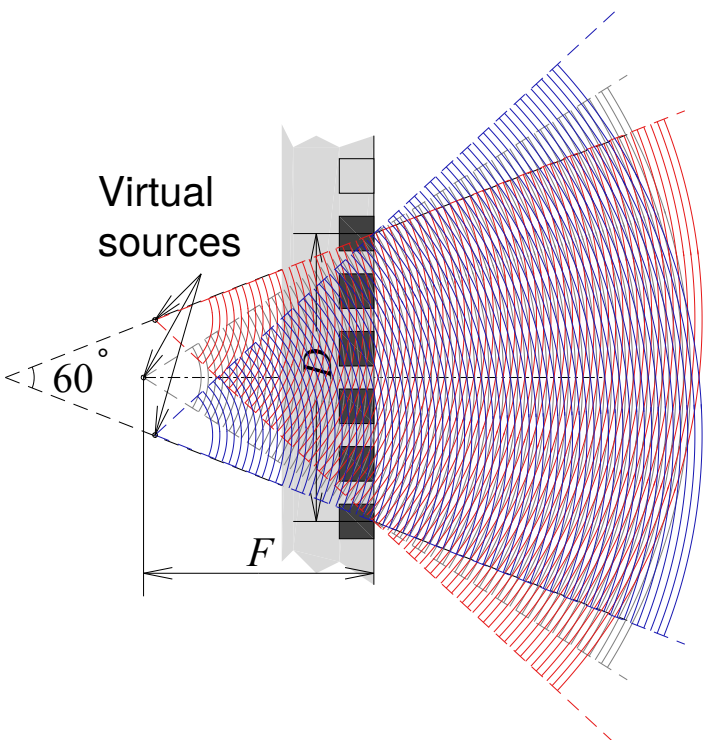

Fig. 2: Synthetic aperture B-mode beam steering with no translation. The concept is illustrated in a plane parallel to the 2-D transducer and is not to scale. The virtual sources are located behind the aperture. $D$ is the active aperture dimension, and $F$ denotes the focal point distance of the middle emission to the the center of the active aperture. The final high resolution B-mode volume is the overlapping region with a $60^{\circ}$ field of view.

mode emission, a low resolution volume was beamformed. After a full transmit cycle, 36 low resolution volumes were coherently added to form the final high resolution volume. After each $B_{M}$ emission, a full volume with a $60^{\circ} \times 60^{\circ}$ fieldof-view could be beamformed. An 8 cycle Hanning-weighted pulse was used for flow emissions and a 2 cycle excitation was used for B-mode emissions.

\section{B. Velocity estimators}

The axial velocity $v_{z}$ estimates were based on the autocorrelation approach [31], and the two transverse velocity components were found by using the TO phase shift estimator and the beamforming procedure described in [13][32].

For each transverse velocity estimate, two TO beams were beamformed along the lines separated spatially by their respective transverse wavelengths $\lambda_{x} / 4$ or $\lambda_{y} / 4$, thereby generating two fields phase-shifted by $90^{\circ}$. The two transverse wavelengths are theoretically given by

$$
\begin{aligned}
& \lambda_{x}(z)=2 \lambda_{z} \frac{z}{d_{x}} \\
& \lambda_{y}(z)=2 \lambda_{z} \frac{z}{d_{y}},
\end{aligned}
$$

where $\lambda_{z}$ is the wavelength of the emitted pulse, $z$ is the axial depth of the beamformed RF-line and $d_{x}, d_{y}$ the distance between the center of the two peaks in the receive apodization in the $x$ - and $y$-directions. The receive apodization profile consists of two rect profiles spanning respectively 8 and 10 elements, with a spacing of 24 elements for $d_{y}$ and 22 elements for $d_{x}$.

The applied 2-D array transducer contains three inactive rows in the $x$-direction due to construction issues. The dimensions of the actual transducer is therefore $35 \times 32$ with the active aperture being $32 \times 32$. Due to the asymmetric geometry of the transducer and different spacing between the TO apodization profiles, two distinct transverse wavelengths must be found and used for velocity estimation in 3-D.

The transverse wavelengths $\lambda_{x}$ and $\lambda_{y}$ were $3.21 \mathrm{~mm}$ and $2.55 \mathrm{~mm}$, respectively, at the depth of $2 \mathrm{~cm}$. The wavelengths were estimated from the TO spatio-temporal frequency spectrum to lower the bias on the estimated velocities [33], and were simulated in Field II [34], [35]. The transverse wavelengths were used to calculate the TO beamforming angles as they were approximately scalable through a linear fit.

The robustness of the velocity estimate is improved by averaging the estimated velocities over the pulse length [32]. The number of emissions per estimate was 16 .

\section{Phase shifting}

The TO method is a phase shift estimator where a fourquadrant inverse tangent operation is used in the calculations. Thus, there is a limitation of the maximum detectable velocity, since the phase range spans from $[-\pi: \pi]$, which translates to $\left[-v_{\max }: v_{\max }\right]$. From [32], the maximum detectable transverse velocities that can be estimated before reaching the aliasing limit are

$$
\begin{aligned}
& v_{x_{\max }}=\frac{\lambda_{x}}{4 k} \frac{f_{p r f}}{N+1} \\
& v_{y_{\max }}=\frac{\lambda_{y}}{4 k} \frac{f_{\text {prf }}}{N+1},
\end{aligned}
$$

where $k$ is the lag used in the autocorrelation and $f_{p r f}$, is the effective pulse repetition frequency between two identical 
transmit events. This phase range can be shifted a maximum of $\pi$ in either direction to increase the maximum detectable velocity without reaching the aliasing limit [36]. In this study the phase range was placed at $\left[-\frac{\pi}{2} ; \frac{3 \pi}{2}\right]$, which translates to a detectable velocity range of $\left[-\frac{1}{2} v_{\max }: \frac{3}{2} v_{\max }\right]$ to match the expected velocities.

\section{Data processing}

The stored data were processed offline. The raw RF data were matched filtered by convolving the signal with the timereversed excitation pulse and Hilbert transformed, before the IQ data were beamformed with the Beamformation Toolbox 3 [37]. In this part, the three velocity components were decoupled, such that one line was beamformed for the axial velocity estimation and two dedicated lines were beamformed for each of the two transverse velocity estimates. In total five unique beamformed lines were used to estimate the 3$\mathrm{D}$ velocity vector for each flow line. For a more extensive description of the employed 3-D TO method, see previous work [20], [21], [38]. Echo cancellation of the beamformed data were performed with two different approaches, depending on the expected tissue motion; in the flow-rig measurement, where no tissue movement was expected, echo canceling was performed by subtracting the mean value from 16 samples from the signal. In cases where pulsating flow was resulting in tissue movement, a frequency energy cut-off based algorithm [39], where an ensemble length of 256 was used to filter out frequency contents from tissue movement in the Fourier domain.

\section{E. Sliding window}

A sliding window in the temporal direction, containing 16 samples, was applied in the off-line processing such that data from the oldest transmit event were replaced by data from the newest transmit event [30]. With this setup, the obtainable frame rate was 1145 frames per second $\left(f_{p r f} /(N+1)\right)$ at $f_{p r f}$ $=12.6 \mathrm{kHz}$, which translates to fully independent estimates every $14 \mathrm{~ms}$.

\section{F. Interpolation}

Since velocity estimation is only performed along the direction of the flow lines, interpolation was performed to produce a color flow map (CFM). Interpolation was done by scan converting the velocities according to their steering angle and then by performing a spline interpolation with boundary conditions outside the drawn mask set to zero velocity. At 20 $\mathrm{mm}$ depth, this corresponded to a lateral spacing between the flow lines of $1.2 \mathrm{~mm}$. The final image had the dimensions of $512 \times 512$ pixels $\left(30 \times 30 \mathrm{~mm}^{2}\right)$. A manual segmentation mask $M$ of the lumen was made based on the B-mode images to determine the flow regions.

\section{G. Angle Estimation}

The processed data provided the velocity components $v_{x}, v_{y}$ and $v_{z}$ along each steered direction (Fig. 3). The direction of the flow, given by the angles $\alpha$ and $\beta$ can be determined as

$$
\begin{aligned}
& \alpha=\sin \frac{v_{z}}{|\mathbf{v}|} \\
& \beta=\arctan \frac{v_{y} /|\mathbf{v}|}{v_{x} /|\mathbf{v}|}=\arctan \frac{v_{y}}{v_{x}},
\end{aligned}
$$

where $|\mathbf{v}|$ is the magnitude of the velocity, $\alpha$ is the rotation around the $y$-axis and $\beta$ the rotation around the $z$-axis. The estimated angles were used to estimate the direction of the flow in the in vivo setup.

\section{H. Flow rate estimation}

The instantaneous volumetric flow rate $Q(t)$ is defined as

$$
Q(t)=\sum_{i} A_{i} v_{i}(t)
$$

where the cross-section of the blood vessel is divided into small areas of size $A_{i}$ each with a velocity component $v_{i}(t)$, propagating perpendicularly to $A_{i}$. In this paper, the index value $i$ for the velocity component was replaced by the pixel values in $\left(x_{j}, y_{k}\right)$ and the cross-sectional area $A$ was the metric area of a pixel size which was constant. The flow rates at each pixel were calculated by multiplying the scan converted out-ofplane velocity component $v_{x}\left(x_{j}, y_{k}\right)$ with the corresponding pixel value for the drawn mask $M\left(x_{j}, y_{k}\right)$ and the crosssectional area $A$. Mask values were either 0 or 1 and constant in time. The flow rate at time $t$ was then

$$
Q(t)=\sum_{j} \sum_{k} A v_{x}\left(x_{j}, y_{k}, t\right) M\left(x_{j}, y_{k}\right),
$$

where the summation ran over all the $j \times k$ pixels in the image.

In the experimental setup, flow rates were estimated based on a beamformed B-mode volume where the plane parallel to the flow transmissions was selected for further processing. The B-mode image was used for manually delineating a mask of the cross sectional vessel. Only flow within this mask was used in the further processing. After the delineation mask was drawn, the flow was processed, scan converted, interpolated and the mask was multiplied to the final estimates. Within this mask, the flow rates, based on the velocity component perpendicular to the plane were calculated as a function of time.

\section{US MEASUREMENT SetuP}

The following section describes the different equipment and parameters used for the phantom and in vivo measurements. Transducer properties and flow estimation parameters are listed in Table I. An illustration of the experimental setup and its coordinate system is shown in Fig. 3. The only difference between the phantom and in vivo measurements was the $f_{p r f}$, which was adjusted in accordance with the actual flow velocities. 


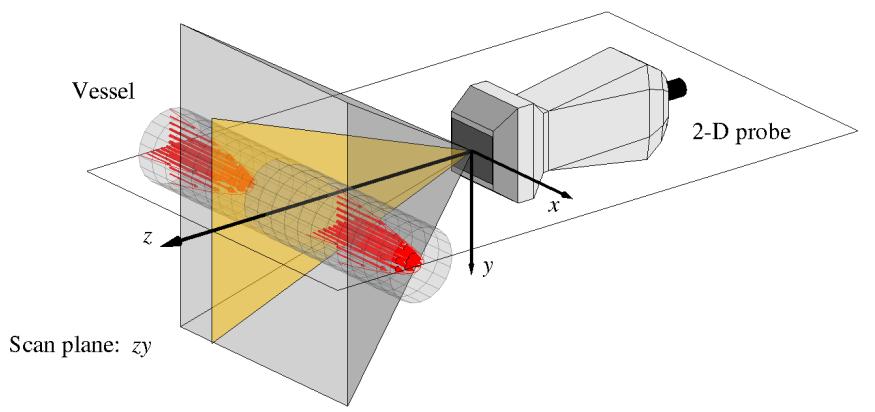

Fig. 3: A 2-D matrix probe was oriented at a $90^{\circ}$ degree beamto-flow angle with the flow moving in the $x$-direction. Flow data (yellow plane) are only obtained for the $z y$-plane, whereas B-mode (gray box) is present in a symmetric volume. The angles spanned are only for the illustration and not to scale.

\section{A. Scanner setup}

The experimental ultrasound scanner SARUS [40] with 1024 channels in receive and transmit was used along with a $3.5 \mathrm{MHz}, 0.3 \mathrm{~mm}$ pitch, $0.278 \mathrm{~mm}$ height $32 \times 32$ element 2-D phased array transducer (Vermon S.A., Tours, France) for data acquisition. See Table I for transducer specifications. The emitted frequency was 3.0 MHz. Data were sampled from all 1024 channels and stored for offline processing on a Linux cluster.

\section{B. Flow-rig}

An in-house built flow-rig system was used to validate flow rate estimates in a steady flow. The flow-rig contained a long inlet of $1.2 \mathrm{~m}$, which ensured that a steady laminar parabolic flow profile was present at the measuring site. Blood mimicking fluid was circulated inside the vessel by a centrifugal pump in a closed loop circuit. At the measuring site, the rubber vessel $(\varnothing=12 \mathrm{~mm})$ was immersed into a water tank containing demineralized water. The volume flow $Q$ was measured with a MAG 1100 flow meter (Danfoss, Hasselager, Denmark). The transducer was placed in a fixture at a $90^{\circ}$ beam-to-flow angle and the fixture was then placed in the water tank and aligned to the center of the vessel. A flow rate of $79.80 \mathrm{~mL} / \mathrm{min}$ translating to a peak velocity of $39 \mathrm{~cm} / \mathrm{s}$ was chosen.

\section{Flow-rig measurements}

The transducer was oriented to acquire 3-D vector flow in a cross sectional scan plane and fixated in a $90^{\circ}$ beam-to-flow angle with a distance of $2.6 \mathrm{~cm}$ from transducer surface to the vessel center. $f_{p r f}$ was scaled to $5 \mathrm{kHz}$ to match with the expected velocity range. The maximum detectable out-ofplane velocity component $v_{x}$, which was expected to have the largest magnitude, was at $2 \mathrm{~cm}$ depth

$$
v_{x_{\text {max }}}=\frac{\lambda_{x}}{4} \frac{f_{\text {prf }}}{N+1}=\frac{0.32 \mathrm{~cm}}{4} \frac{5000 \mathrm{~s}^{-1}}{10+1}=36 \mathrm{~cm} / \mathrm{s} .
$$

With the $\pi / 2$ phase shift, this translates to a detectable velocity range of $[-18 ; 55] \mathrm{cm} / \mathrm{s}$.

\section{Pulsatile flow pump}

For the phantom measurements a predefined pulsatile carotid flow profile was generated with a flow pump (CompuFlow 1000 System, Shelley Medical Imaging Technologies, Ontario, Canada) circulating blood mimicking fluid with a backscattering coefficient equivalent to blood cells into a straight C-flex $(\mathrm{c}=1550 \mathrm{~m} / \mathrm{s})$ tube $(\oslash=8 \mathrm{~mm}, 22 \mathrm{~cm}$ long, and $0.8 \mathrm{~mm}$ thick), which was surrounded by a tissue mimicking material. The attenuation of the tissue mimicking material was $0.5 \mathrm{~dB} /(\mathrm{cm} \cdot \mathrm{MHz})$, and the speed of sound was $1540 \mathrm{~m} / \mathrm{s}$, which complies with previous reported properties [41]. With the included CompuFlow 1000 software, a carotid flow profile was generated with a cycle time of $0.84 \mathrm{~s}$ and a flow rate of $2.57 \mathrm{~mL} / \mathrm{stroke} \pm 3 \%$ as specified by the manufacturer.

\section{E. Flow pump measurements}

The transducer was oriented to acquire 3-D vector flow in a cross sectional scan plane and fixated in a $90^{\circ}$ beam-to-flow angle with a distance of $1.9 \mathrm{~cm}$ from the transducer surface to the vessel center. $f_{p r f}$ was $5 \mathrm{kHz}$ and a $\pi / 2$ phase shift was applied to comply with the expected velocity range.

\section{F. FDA limits}

Intensity measurements were performed to ensure compliance with current FDA intensity regulations [42]. A setup with an Onda HGL-0400 hydrophone (Onda Corp., Sunnyvale, CA) connected to an Onda ATH-2000 attenuator connected to an Onda AH-2000 pre-amplifier was placed in a water tank to measure the pressure field generated in the plane where the highest intensities were expected, i.e the $z y$-plane where flow emissions were transmitted [43]. At every specified point in the $z y$-plane, the pressure field was recorded for the complete transmit sequence (B-mode and flow) using the approach described in [43], at $f_{p r f}=100 \mathrm{~Hz}$. The derated mechanical index (MI) was 1.14 and the system pulse repetition frequency $f_{\text {prf }}$ was scaled to $12.6 \mathrm{kHz}$ for the in vivo measurements to obtain $I_{\text {spta.3 }}=439 \mathrm{~mW} / \mathrm{cm}^{2}$ which is below FDA limits.

\section{G. Clinical setup for the in vivo measurements}

The in vivo measurement was performed on a healthy 27 year old male, who had been resting for $15 \mathrm{~min}$ before the measurements to ensure steady state flow. The scans were performed by an experienced radiologist (CE). Prior to the experimental scans and prior to the MRI scans, a 1-D spectral Doppler reference measurement was made. The reference measurements were made with a linear 5.2 MHz probe (9032, BK Ultrasound, Herlev, Denmark) and a commercial scanner (BK 5000, BK Ultrasound, Herlev, Denmark). Properties for the in vivo sequence are summarised in Table I.

The system pulse repetition frequency $f_{p r f}$ was $12.6 \mathrm{kHz}$ in the in vivo study to yield a higher detectable velocity range, especially for the $v_{x}$-component, which was $[-45 ; 137] \mathrm{cm} / \mathrm{s}$ at $2 \mathrm{~cm}$ depth. Cross sectional measurements were conducted $2-3 \mathrm{~cm}$ before the bifurcation in the common carotid artery. 


\section{MRI SCANS}

\section{A. MRI experimental setup}

A $1.5 \mathrm{~T}$ whole body scanner (Avanti, Siemens, Erlangen, Germany) was used for estimation of through-plane velocities in a cross sectional view of the right common carotid artery. A retrospective electrocardiography (ECG) gated phase contrast sequence in combination with a head and a neck matrix coil were used to estimate through-plane velocities in a plane. The sequence had a repetition time of $42 \mathrm{~ms}$, echo time $3 \mathrm{~ms}$, flip angle $20^{\circ}$, pixel resolution of $1.1 \times 1.1 \mathrm{~mm}$ in an image of size $216 \times 256$ pixels, slice thickness $5 \mathrm{~mm}$, and a maximum velocity encoding of $\pm 1.0 \mathrm{~m} / \mathrm{s}$. Estimates were retrieved from 210 heartbeats. An anatomical image with similar resolution was acquired parallel to the applied flow sequence. Three similar measurements were performed such that a standard deviation on MRI results could be calculated. The measurements were performed $2-3 \mathrm{~cm}$ before the bifurcation in the common carotid artery to comply with the in vivo 3-D ultrasound measurement. The volunteer had been resting $15 \mathrm{~min}$ before the MRI examination. Prior to the MRI examination, a spectral Doppler measurement with similar equipment as described in III-G was performed for reference.

\section{B. MRI processing}

Three velocity data sets were acquired and processed offline. Each data set contained anatomical and through-plane velocity information in an entire plane from 50 different time instances in the cardiac cycle. A data set was processed by reading the stored DICOM files into MATLAB and adding up all the 50 temporal frames to create a combined anatomical intensity map, ( Fig. 4a), to suppress noise and enhance vessel regions. Based on the intensity map, a binary image was created with a threshold of $35 \%$ of the maximum intensity to segment out vessel regions (Fig. 4b). A morphological opening operation was used to remove objects less than $20 \mathrm{~mm}^{2}$ from the binary image. A boundary detection algorithm was run on the binary image, to identify the perimeter of the remaining object. Based on the identified regions of interest (ROI), a manual selection of the region, where the cross sectional common carotid artery was expected was performed (Fig. 4c), which created a binary ROI mask. Since anatomical data and flow data were acquired in parallel, the selected mask was applied on the flow data (Fig. 4d), such that all pixels inside the ROI were used to estimate peak velocities and temporal flow rates as stated in eq. (6). The described procedure was applied to all three acquisitions.

\section{RESULTS}

\section{A. Flow-rig measurements}

A total of $15.8 \mathrm{~s}$ of data were acquired. Due to an ensemble length of 16 , the temporal flow rate estimates were divided into sections of similar size, and the mean from each section was calculated. The standard deviation was calculated from the mean of all the sections. This gave an estimated flow rate of $82.1 \pm 2.8 \mathrm{~L} / \mathrm{min}$ compared with the expected 79.80 $\mathrm{L} / \mathrm{min}$. The delineated area was $114 \mathrm{~mm}^{2}$ compared with the theoretical $113 \mathrm{~mm}^{2}$.

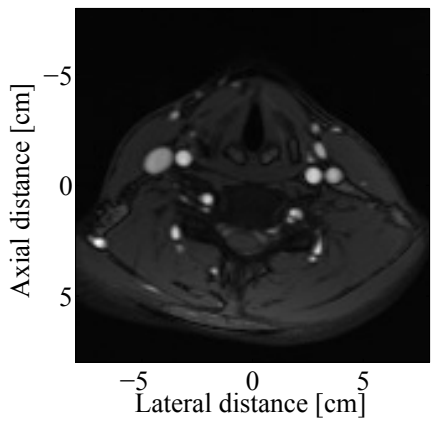

(a)

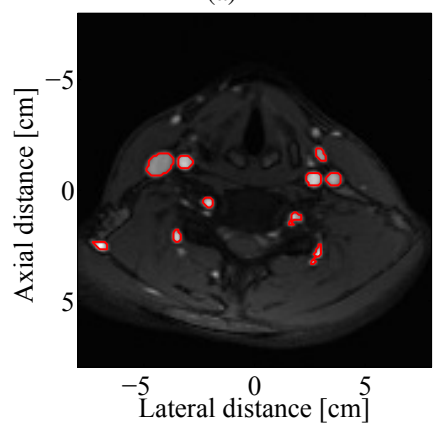

(c)

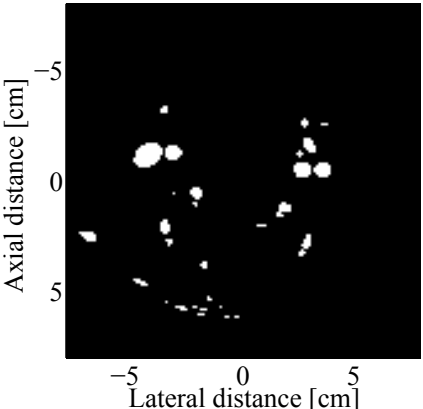

(b)

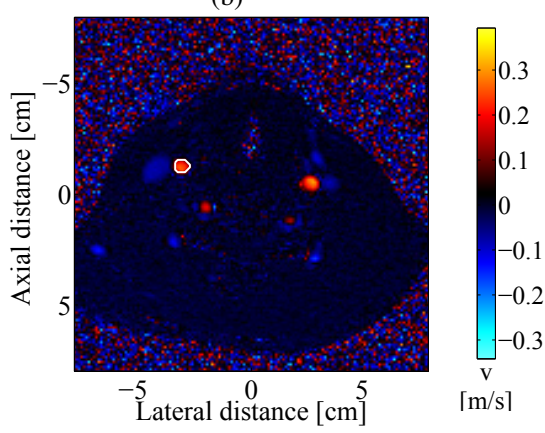

(d)
Fig. 4: a) Summed anatomical intensity map. b) Binary anatomical image based on a 35\% threshold of the maximum intensity c) Identified ROI after morphological opening and boundary detection, which was presented for the radiologist for identification of the right common carotid artery. d) Mean velocity image with the identified ROI superimposed on top (white ring). Pixels inside this ROI were used for flow rate and peak velocity estimation.

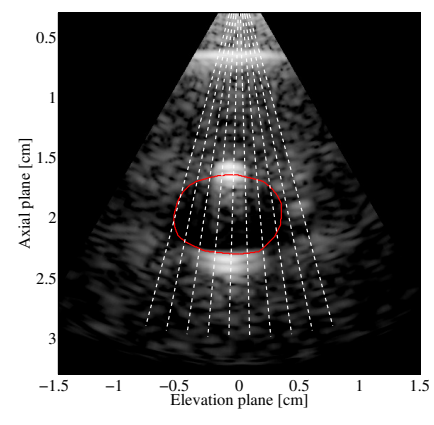

(a)

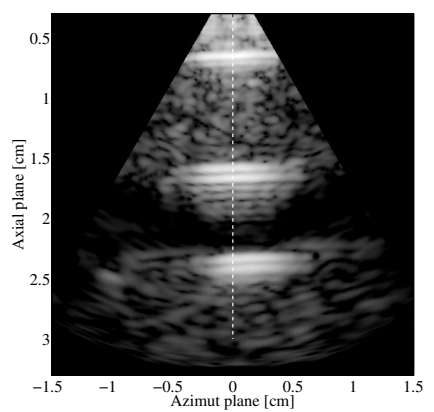

(b)
Fig. 5: a) Cross sectional image plane of the straight-vessel phantom with the drawn mask (red curve) and the flow emissions (dotted lines). b) Longitudinal scan plane orthogonal to a). The images are planes taken out from the beamformed volume and are shown at a $50 \mathrm{~dB}$ dynamic range.

\section{B. Flow pump measurements}

A total of $18.9 \mathrm{~s}$ of data were acquired and data processing was made similar to the flow-rig measurement. The delineated mask in the cross sectional vessel is shown in Fig. 5.

The estimated temporal flow rates were used to automatically estimate the average time between each cycle with an autocorrelation routine. A cycle time of $838 \mathrm{~ms}$, which 


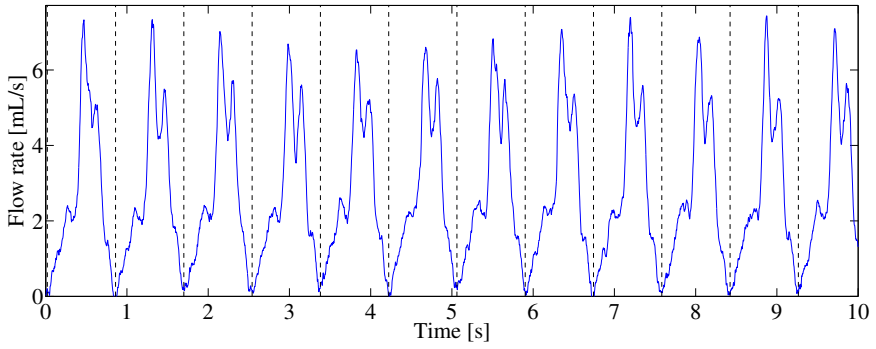

(a)

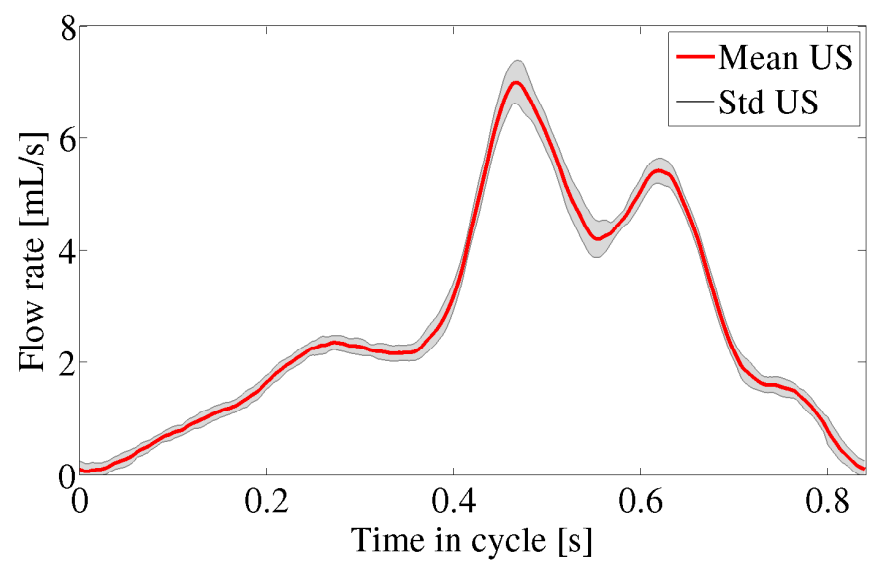

(b)

Fig. 6: a) Temporal volumetric flow rate based on the delineated cross sectional vessel area, and the velocity component perpendicular to the plane. The dotted lines illustrate the start and end of the estimated cycle. b) Mean cycle flow rate (red curve) \pm one standard deviation (grey area) estimated from 22 coherently aligned flow rate estimates.

translates to 382 velocity estimates per cycle and 22 cycles in total, was observed. This was in agreement with the software specified $840 \mathrm{~ms}$ cycle time.

The data set was divided into 22 cycles, which could be coherently aligned. The temporal volumetric flow rates with the cycle division are shown for the first $10 \mathrm{~s}$ of acquisition ( Fig. 6a). A flow rate of $2.68 \pm 0.04 \mathrm{~mL} /$ stroke was found from the coherently aligned 22 cycles (see Fig. 6b) compared with the $2.57 \pm 0.08 \mathrm{~mL} /$ stroke specified by the manufacture. The delineated vessel area was $48.4 \mathrm{~mm}^{2}$ compared with the expected area of $50.3 \mathrm{~mm}^{2}$ calculated from the specified vessel radius.

\section{In vivo US measurements}

For the in vivo measurements, $7.5 \mathrm{~s}$ of data were recorded. Data were processed as described in SectionV-B. A heart cycle time of $1.17 \mathrm{~s}$ gave an estimated heart rate of 51 beats/min, which translates to 1.345 velocity estimates per cycle. In total 6 complete heart cycles were identified, and the vessel center was located at a depth of $1.5 \mathrm{~cm}$, as shown in Fig. 7a. Due to the short acquisition time, it was expected that the temporal flow rates during each cycle could be coherently aligned. Alignment of the flow rates are shown in Fig. 7b.

Fig. 7c, shows the aligned mean flow rate. A flow rate of 6.5 $\pm 0.6 \mathrm{~mL} /$ stroke, translating to $333 \pm 31 \mathrm{~mL} / \mathrm{min}$, was seen for the 6 cycles and the vessel area was estimated to $62 \mathrm{~mm}^{2}$. The maximum peak systolic velocity magnitude for each cycle was $99 \pm 5 \mathrm{~cm} / \mathrm{s}$ for 3-D VFI compared with a value of 107 $\pm 2 \mathrm{~cm} / \mathrm{s}$ obtained with spectral Doppler techniques prior to the measurements (Fig. 7d).

Since 3-D vector flow data were present, the beam-toflow angle $\alpha$ and the flow rotation angle $\beta$ were calculated throughout the coherently added cycles. The angles were calculated from the similar ROI used for estimating peak velocities. A flow perpendicular to the scan plane (i.e. a $90^{\circ}$ beam-to-flow angle) would result in $\alpha=0^{\circ}$ and $\beta=0^{\circ}$. The temporal flow directions are seen in Fig. 8 with the mean precession values $\alpha=2.4^{\circ} \pm 0.9^{\circ}$ and $\beta=-29.1^{\circ} \pm 0.8^{\circ}$. The angulation of the transducer is also illustrated in a 3-D vector flow map during the end-diastole (Fig. 9a), but mostly during the peak-systole (Fig. 9b.)

\section{MRI measurements}

Data from the three MRI measurements were processed off-line as described in IV-B. The mean vessel area of the right common carotid artery was $41.7 \mathrm{~mm}^{2}$ and temporal flow rates and peak velocities are shown in Figs. $7 \mathrm{c}$ and $7 \mathrm{~d}$. The mean flow rate was $7.44 \mathrm{~mL} / \mathrm{stroke} \pm 0.04 \mathrm{~mL} / \mathrm{stroke}$ and the peak velocity was $77 \pm 1 \mathrm{~cm} / \mathrm{s}$ compared with $101 \pm 1 \mathrm{~cm} / \mathrm{s}$ obtained with spectral Doppler before the measurements.

The heart cycle lasted $1.29 \mathrm{~s}$, which translates to a flow rate of $346 \pm 2 \mathrm{~mL} / \mathrm{min}$.

\section{DISCUSSION}

Both phantom measurements showed that the proposed method for flow rate estimation provided a high relative precision of $3.4 \%$ and $1.5 \%$ for constant and pulsatile flow, respectively. Furthermore a small bias of $2.9 \%$ for the flow-rig measurement and $4.3 \%$ for the pulsating flow pump measurement was seen.

The estimated peak velocities for MRI and 3-D vector flow were similar, with an exception in peak systole. In peak systole US estimates were significantly higher than the MRI measurements. Also in the early diastole, 3-D vector flow US estimated significantly lower than MRI. The peak velocities estimated with US were angle independent and derived from the 3-D velocity vector, whereas MRI peak velocities were entirely based on the through plane component. With MRI the highest peak velocities were expected when the common carotid artery was aligned exactly perpendicular to the imaging scan plane. This could not be ensured in the MRI images, but based on the volumetric anatomical data it was possible to estimate the rotation of the vessel compared with the actual scan plane in the MRI examination. The rotation was estimated to $14^{\circ}$, which results in an increased angle corrected peak velocity of $3 \%$. Thus, the underestimation in peak velocities found with MRI in this study was not expected to be due to the vessel alignment.

The reduced fluctuation in estimated peak velocities with MRI compared with 3-D vector flow, can also be due to the difference in spatial and temporal resolution. The spatial resolution of $1.1 \mathrm{~mm}^{2} /$ pixel for MRI, results in velocity estimates 


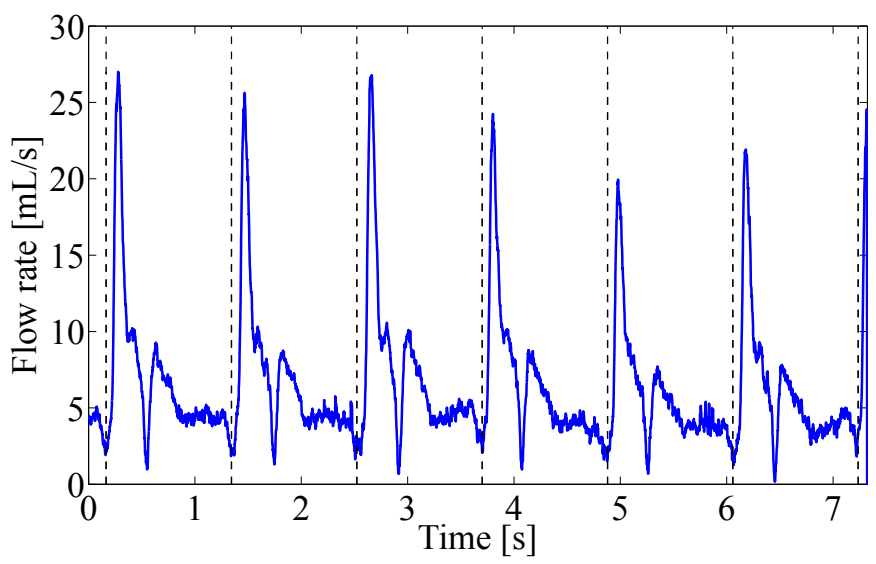

(a)

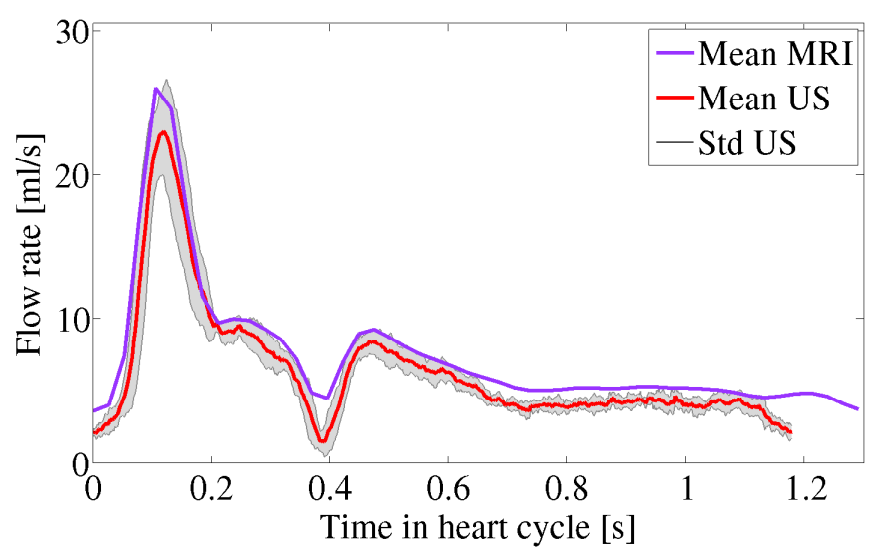

(c)

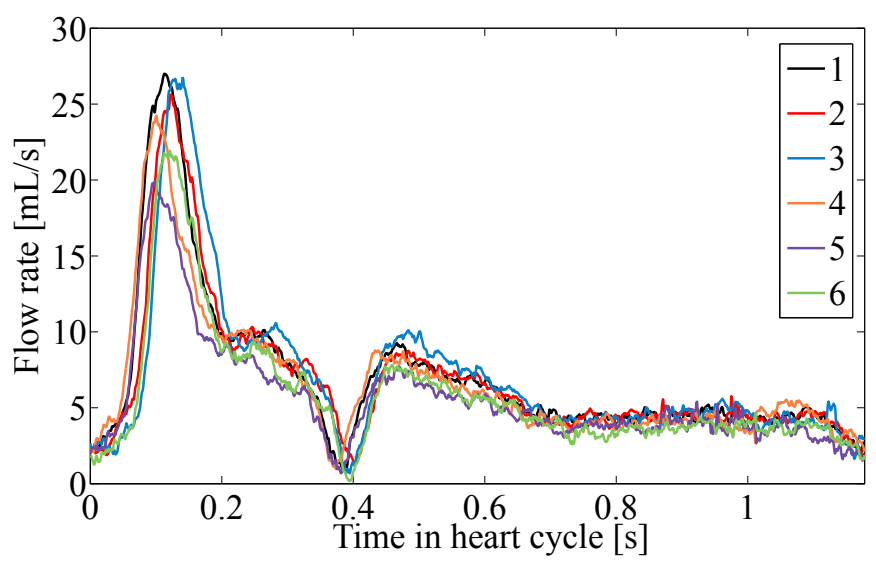

(b)

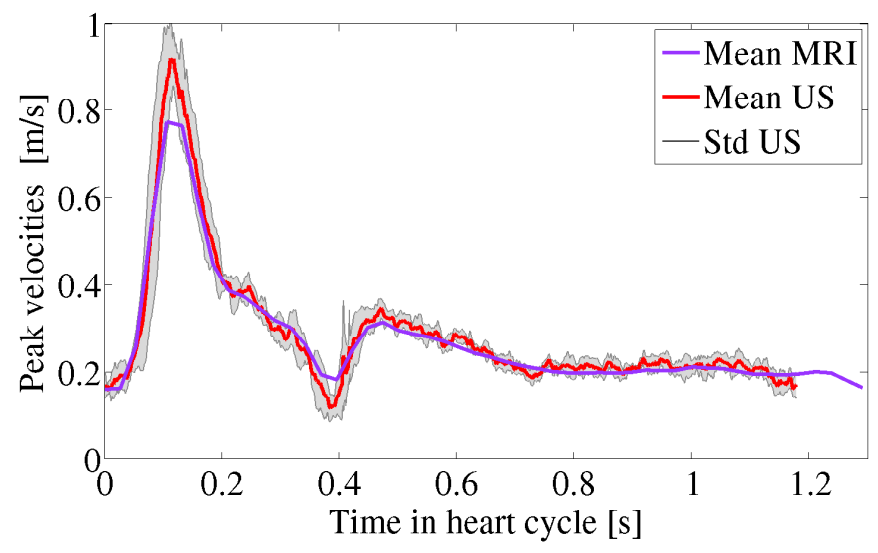

(d)

Fig. 7: a) Estimated flow rates (blue curve) with the applied heart cycle division (dotted lines). b) Estimated flow rates of the 6 coherently aligned heart cycles. c) Mean volumetric flow rate throughout a heart cycle. MRI (purple curve) and US (red curve \pm one standard deviation). d) Peak through plane velocities for MRI (purple curve) and US (red curve \pm one standard deviation).

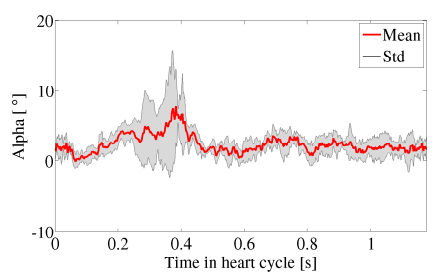

(a)

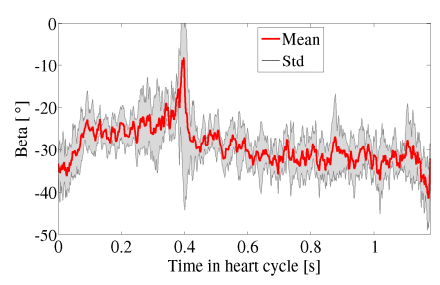

(b)
Fig. 8: a) Estimated in vivo beam-to-flow angle $\alpha$. b) Estimated in vivo flow direction $\beta$.

averaged within a relatively large area compared with both 3D VFI and spectral Doppler estimates. This smoothing effect is further enhanced by the long acquisition of 210 heart beats, where very small patient movements of only $0.5 \mathrm{~mm}$ will result in a shift in the velocity estimates by one pixel.

A fair comparison between MRI and the 3-D vector flow method may be by estimating flow rates. This measure is angle independent, as long as the cross sectional vessel area is present and the velocities perpendicular to this plane are estimated, which was the case for both MRI and US.

A high precision was found for the US estimated flow rates of $9.1 \%$ even though data from only 6 completed cycles were present. MRI had a much higher precision of $0.5 \%$, from 3 acquisitions each containing the mean from 210 heart beats. The actual estimated mean flow rates with US and MRI deviated by $15 \%$ when measuring in $\mathrm{mL} / \mathrm{stroke}$, but only by $3.9 \%$ when using $\mathrm{mL} / \mathrm{min}$. However, the results were obtained based on two different cross-sectional vessel areas of $62 \mathrm{~mm}^{2}$ for US and $42 \mathrm{~mm}^{2}$ for MRI.

It is difficult to tell, if the vessel was delineated correctly in both modalities, since the ground-truth could not be obtained, and also, since the vessel could intersect with the respective scan-plane at different angles. Future work should investigate the effect of using a time-varying vessel mask, which both tracks the displacement and the expansion/contraction of the vessel.

Even though the volunteer had undergone the same resting procedure prior to MRI and US measurements, the flow dynamics were expected to fluctuate. However, the spectral Doppler results obtained prior to both measurements were consistent $(\mathrm{PS}=101.3 \pm 1.2 \mathrm{~cm} / \mathrm{s}$ before MRI and 106.8 


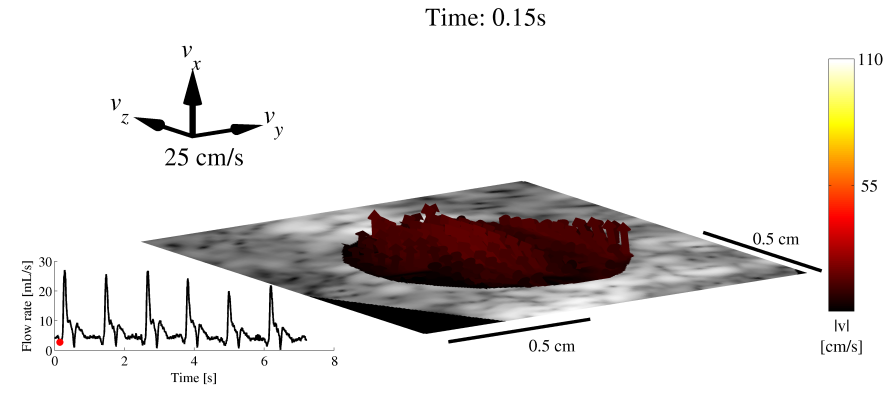

(a) 3-D vector velocities at the end-diastole

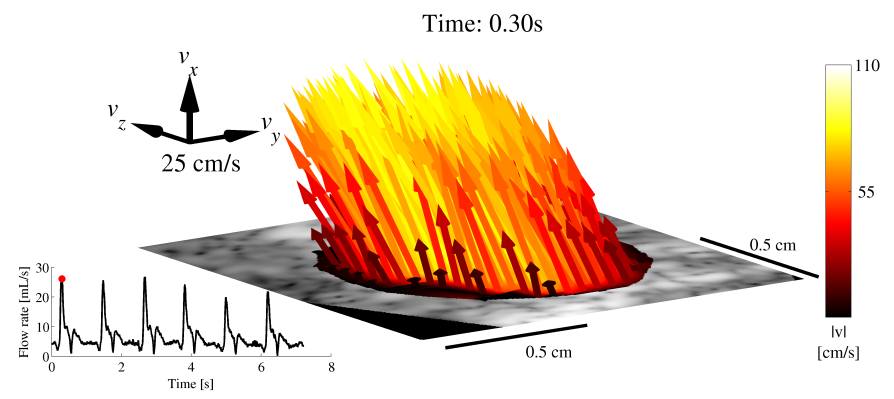

(b) 3-D vector velocities at the peak-systole

Fig. 9: 3-D vector flow from the common carotid artery in end-diastole a) and in peak-systole b). The coloured arrows depict the direction of the flow and its magnitude. The scan was not exactly performed perpendicular to the vessel, which is revealed in b) where a significant $v_{y}$ velocity component is present. The bottom-left graphs show the flow rate at the time instance in the heart cycle indicated by the the red dot.

$\pm 1.7 \mathrm{~cm} / \mathrm{s}$ before US) and the heart cycle time was 1.29 $\mathrm{s}$ during MRI measurements and $1.17 \mathrm{~s}$ while obtaining the US data. The fluctuation in flow dynamics between the two measurements was therefore expected to be small. Based on the two spectral Doppler measurements, a relative difference in peak velocities between US and MRI of $\sim 5 \%$ should be expected. Moreover, due to the two estimated cycle times a relative difference in flow rates of $\sim 10 \%$ could be expected, when measuring in units of $\mathrm{mL} /$ stroke.

Sampling data from a total of 1024 channels can seem impractical, but currently, these are the order of 2-D fully populated matrix probes. Even significantly larger matrix array are currently available for commercial use, as the 9.212 element fully sampled phased matrix array X6-1 PureWave xMATRIX probe from Phillips (Eindhoven, Netherlands).

In this study focused emission were used for vector flow estimation. The reason being, that focused emissions provide a higher signal-to-noise ratio (SNR) compared to plane waves or diverging waves at the focal point. Future work should explore the potential of combining TO with plane waves or diverging wave transmission, which can be used to estimate 3 -D vector flow in a plane or in the entire volume. Recent work has been reported in the literature for estimating volumetric 3-D flow using a 2-D matrix probe for plane waves combined with vector Doppler [44], and synthetic aperture directional beamforming combined with diverging waves [45]. For such of transmissions, the transmitted energy will be distributed into a larger region compared with focused emissions. This results in a poorer SNR but may be compensated by the several transmit events overlapping each other.

Among the competing vector flow techniques is Vector Doppler. Vector Doppler techniques exploit that the triangulation of individual axial velocity estimates achieved from plane waves steered in different directions, can provide 2-D or 3-D VFI. The methods use a phase shift estimator, where the aliasing limit is proportional to the axial wavelength. As a consequence of this, transmit sequences used for vector Doppler methods are restricted to only contain few unique flow emissions to avoid reaching the aliasing limit, compared with a sequence used for TO. However, this can be counterbalanced by the axial estimator usually being more robust than the TO estimator.

As the temporal flow rate is $14 \mathrm{~ms}$ for the velocity estimates provided in this study, it is expected that the method is capable of capturing complex flow and vortices, as their lifespan is in the order of 100-200 ms [46].

\section{CONCLUSION}

We have proposed a method for high frame rate 3-D vector flow estimation in a plane. The purpose of the method was to estimate angle independent peak velocities and flow rates. Two phantom measurements were performed to validate the estimated flow rates, which were both within $5 \%$ of the expected values.

Furthermore, we presented the first quantitative in vivo comparison between high frame rate 3-D vector flow estimated with US and through plane velocities obtained from MRI. The results showed that a precision of $9.1 \%$ from only 6 heart cycles could be obtained for US estimated flow rates, and a $5 \%$ negative bias on peak velocities compared with spectral Doppler measurements. The measured flow rates in US and MRI only deviated $3.9 \%$ when translating to $\mathrm{mL} / \mathrm{min}$. Whether which, or any, of the methods were overestimating or underestimating in the in vivo measurements were not clarified in this study. A larger clinical study is expected to be carried out for validating the performance of 3-D VFI using US compared with MRI.

\section{ACKNOWLEDGMENT}

This work was supported by grant 82-2012-4 from the Danish Advanced Technology Foundation and by BK Ultrasound Aps.

\section{REFERENCES}

[1] WHO, "Global status report on noncommunicable diseases 2010," 2011.

[2] P. A. Picot and P. M. Embree, "Quantitative volume flow estimation using velocity profiles," IEEE Trans. Ultrason., Ferroelec., Freq. Contr., vol. 41, pp. 340-345, 1994.

[3] J. Jensen, J. B. Olesen, M. B. Stuart, P. M. Hansen, M. B. Nielsen, and J. A. Jensen, "Vector velocity volume flow estimation: Sources of error and corrections applied for arteriovenous fistulas," Ultrasonics, vol. 70, pp. 136-146, 2016. 
[4] L. N. Bohs and G. E. Trahey, "A novel method for angle independent ultrasonic imaging of blood flow and tissue motion," IEEE Trans. Biomed. Eng., vol. 38, pp. 280-286, 1991.

[5] S. I. Nikolov and J. A. Jensen, "Velocity estimation using synthetic aperture imaging," in Proc. IEEE Ultrason. Symp., 2001, pp. 1409-1412.

[6] J. A. Jensen and S. I. Nikolov, "Directional synthetic aperture flow imaging," IEEE Trans. Ultrason., Ferroelec., Freq. Contr., vol. 51, pp. 1107-1118, 2004

[7] C. A. Villagomez-Hoyos, M. B. Stuart, K. L. Hansen, M. B. Nielsen, and J. A. Jensen, "Accurate angle estimator for high frame rate 2-D vector flow imaging," IEEE Trans. Ultrason., Ferroelec., Freq. Contr., vol. 63, no. 6, pp. 842-853, 2016.

[8] J. Udesen, F. Gran, K. L. Hansen, J. A. Jensen, C. Thomsen, and M. B. Nielsen, "High frame-rate blood vector velocity imaging using plane waves: simulations and preliminary experiments," IEEE Trans. Ultrason., Ferroelec., Freq. Contr., vol. 55, no. 8, pp. 1729-1743, 2008.

[9] B. Y. Yiu, S. S. Lai, and A. C. Yu, "Vector projectile imaging: timeresolved dynamic visualization of complex flow patterns." Ultrasound Med. Biol., vol. 40, no. 9, pp. 2295-2309, sept 2014.

[10] S. Fadnes, I. K. Ekroll, S. A. Nyrnes, H. Torp, and L. Løvstakken, "Robust angle-independent blood velocity estimation based on dualangle plane wave imaging," IEEE Trans. Ultrason., Ferroelec., Freq. Contr., vol. 62, no. 10, pp. 1757-1767, October 2015.

[11] F. Mehregan, F. Tournoux, S. Muth, P. Pibarot, R. Rieu, G. Cloutier, and D. Garcia, "Doppler vortography: a color doppler approach for quantification of the intraventricular blood flow vortices," Ultrasound Med. Biol., vol. 40, no. 1, pp. 210-221, January 2014.

[12] B. Dunmire, K. W. Beach, K.-H. Labs., M. Plett, and D. E. Strandness, "Cross-beam vector Doppler ultrasound for angle independent velocity measurements," Ultrasound Med. Biol., vol. 26, pp. 1213-1235, 2000.

[13] J. A. Jensen and P. Munk, "A new method for estimation of velocity vectors," IEEE Trans. Ultrason., Ferroelec., Freq. Contr., vol. 45, pp. 837-851, 1998.

[14] M. Lenge, A. Ramalli, P. Tortoli, C. Cachard, and H. Liebgott, "Planewave transverse oscillation for high-frame-rate 2-D vector flow imaging," IEEE Trans. Ultrason., Ferroelec., Freq. Contr., vol. 62, no. 12, pp. 2126-2137, December 2015.

[15] I. A. Hein, "Triple-beam lens transducers for three-dimensional ultrasonic fluid flow estimation," IEEE Trans. Ultrason., Ferroelec., Freq. Contr., vol. 42, pp. 854-869, 1995.

[16] C. H. Seo and J. T. Yen, "64 x 64 2-D array transducer with row-column addressing," in Proc. IEEE Ultrason. Symp., vol. 1, 2006, pp. 74-77.

[17] A. Sampaleanu, P. Zhang, A. Kshirsagar, W. Moussa, and R. Zemp, "Top-orthogonal-to-bottom-electrode (TOBE) CMUT arrays for 3-D ultrasound imaging." IEEE Trans. Ultrason., Ferroelec., Freq. Contr. vol. 61, no. 2, pp. 266-276, 2014.

[18] B. Savord and R. Solomon, "Fully sampled matrix transducer for real time 3D ultrasonic imaging," in Proc. IEEE Ultrason. Symp., vol. 1, 2003, pp. 945-953.

[19] S. Holbek, M. Pihl, C. Ewertsen, M. Nielsen, and J. A. Jensen, "3-D velocity estimation for two planes in vivo," in Proc. IEEE Ultrason. Symp., 2014, pp. 1706-1709.

[20] M. J. Pihl and J. A. Jensen, "A transverse oscillation approach for estimation of three-dimensional velocity vectors. Part I: Concept and simulation study," IEEE Trans. Ultrason., Ferroelec., Freq. Contr., vol. 61, pp. 1599-1607, 2014.

[21] M. J. Pihl, M. B. Stuart, B. G. Tomov, M. F. Rasmussen, and J. A. Jensen, "A transverse oscillation approach for estimation of threedimensional velocity vectors. Part II: Experimental validation," IEEE Trans. Ultrason., Ferroelec., Freq. Contr., vol. 51, no. 10, pp. 16081618,2014

[22] J. Provost, C. Papadacci, J. E. Arango, M. Imbault, M. Fink, J. L. Gennisson, M. Tanter, and M. Pernot, "3-D ultrafast ultrasound imaging in vivo," Phys. Med. Biol., vol. 59, no. 19, pp. L1-L13, 2014.

[23] M. Markl, A. Harloff, T. A. Bley, M. Zaitsev, B. Jung, E. Weigang, M. Langer, J. Hennig, and A. Frydrychowicz, "Time-resolved 3D MR velocity mapping at $3 \mathrm{~T}$ : improved navigator-gated assessment of vascular anatomy and blood flow." Journal of magnetic resonance imaging, vol. 25 , no. 4, pp. 824-831, 2007.

[24] K. L. Hansen, J. Udesen, C. Thomsen, J. A. Jensen, and M. B. Nielsen, "In vivo validation of a blood vector velocity estimator with MR angiography," IEEE Trans. Ultrason., Ferroelec., Freq. Contr., vol. 56, no. 1, pp. 91-100, 2009.

[25] A. Harloff, T. Zech, F. Wegent, C. Strecker, C. Weiller, and M. Markl, "Comparison of blood flow velocity quantification by 4D flow MR imaging with ultrasound at the carotid bifurcation," Am. J. Neuroradiol., vol. 34, pp. 1407-1413, 2013.
[26] J. Eriksson, C. Carlhall, P. Dyverfeldt, J. Engvall, A. Bolger, and T. Ebbers, "Semi-automatic quantification of 4D left ventricular blood flow," Journal of Cardiovascular Magnetic Resonance, vol. 12, no. 1, p. 9, 2010.

[27] S. Holbek, M. J. Pihl, C. Ewertsen, M. B. Nielsen, and J. A. Jensen, "In vivo 3-D vector velocity estimation with continuous data," in Proc. IEEE Ultrason. Symp., 2015, pp. 1-4.

[28] S. I. Nikolov and J. A. Jensen, "In-vivo synthetic aperture flow imaging in medical ultrasound," IEEE Trans. Ultrason., Ferroelec., Freq. Contr., vol. 50, no. 7, pp. 848-856, 2003.

[29] M. Tanter, J. Bercoff, L. Sandrin, and M. Fink, "Ultrafast compound imaging for 2-D motion vector estimation: application to transient elastography," IEEE Trans. Ultrason., Ferroelec., Freq. Contr., vol. 49, pp. 1363-1374, 2002

[30] S. I. Nikolov, K. Gammelmark, and J. A. Jensen, "Recursive ultrasound imaging," in Proc. IEEE Ultrason. Symp., vol. 2, 1999, pp. 1621-1625.

[31] C. Kasai, K. Namekawa, A. Koyano, and R. Omoto, "Real-Time TwoDimensional Blood Flow Imaging using an Autocorrelation Technique," IEEE Trans. Son. Ultrason., vol. 32, pp. 458-463, 1985.

[32] J. A. Jensen, "A new estimator for vector velocity estimation," IEEE Trans. Ultrason., Ferroelec., Freq. Contr., vol. 48, no. 4, pp. 886-894, 2001.

[33] J. A. Jensen, A. H. Brandt, and M. B. Nielsen, "Convex array vector velocity imaging using transverse oscillation and its optimization," IEEE Trans. Ultrason., Ferroelec., Freq. Contr., vol. 62, no. 12, pp. $2043-$ 2053, 2015.

[34] J. A. Jensen, "Field: A program for simulating ultrasound systems," Med. Biol. Eng. Comp., vol. 10th Nordic-Baltic Conference on Biomedical Imaging, Vol. 4, Supplement 1, Part 1, pp. 351-353, 1996.

[35] J. A. Jensen and N. B. Svendsen, "Calculation of pressure fields from arbitrarily shaped, apodized, and excited ultrasound transducers," IEEE Trans. Ultrason., Ferroelec., Freq. Contr., vol. 39, pp. 262-267, 1992.

[36] J. A. Jensen, Estimation of Blood Velocities Using Ultrasound: A Signal Processing Approach. New York: Cambridge University Press, 1996.

[37] J. M. Hansen, M. C. Hemmsen, and J. A. Jensen, "An object-oriented multi-threaded software beamformation toolbox," in Proc. SPIE Med. Imag., vol. 7968, March 2011, pp. 79680Y-1-79680Y-9.

[38] M. J. Pihl and J. A. Jensen, "3D vector velocity estimation using a 2D phased array," in Proc. IEEE Ultrason. Symp., 2011, pp. 430-433.

[39] C. A. Villagomez-Hoyos, "Synthetic aperture vector flow imaging," Ph.D. dissertation, Technical University of Denmark, 2016. [Online]. Available: http://findit.dtu.dk/en/catalog/2347162876

[40] J. A. Jensen, H. Holten-Lund, R. T. Nilsson, M. Hansen, U. D. Larsen, R. P. Domsten, B. G. Tomov, M. B. Stuart, S. I. Nikolov, M. J. Pihl, Y. Du, J. H. Rasmussen, and M. F. Rasmussen, "SARUS: A synthetic aperture real-time ultrasound system," IEEE Trans. Ultrason., Ferroelec., Freq. Contr., vol. 60, no. 9, pp. 1838-1852, 2013.

[41] M. O. Culjat, D. G. Oldenberg, P. T. Ewari, and R. S. Singh, "A review of tissue substitutes for ultrasound imaging," Ultrasound Med. Biol., vol. 36 , no. 6 , pp. $861-873,2010$.

[42] FDA, "Information for manufacturers seeking marketing clearance of diagnostic ultrasound systems and transducers," Center for Devices and Radiological Health, United States Food and Drug Administration, Tech. Rep., 2008.

[43] J. A. Jensen, M. F. Rasmussen, M. J. Pihl, S. Holbek, C. A. VillagomezHoyos, D. P. Bradway, M. B. Stuart, and B. G. Tomov, "Safety assessment of advanced imaging sequences, I: Measurements," IEEE Trans. Ultrason., Ferroelec., Freq. Contr., vol. 63, no. 1, pp. 110-119, 2016.

[44] M. Correia, J. Provost, M. Tanter, and M. Pernot, "In-vivo 4D ultrafast vector flow imaging: quantitative assessment of arterial blood flow," in Proc. IEEE Ultrason. Symp., 2016, pp. 1-4.

[45] C. A. Villagomez-Hoyos, S. Holbek, M. B. Stuart, and J. A. Jensen, "High frame rate synthetic aperture 3D vector flow imaging," in Proc. IEEE Ultrason. Symp., 2016, pp. 1-4.

[46] D. H. Evans, J. A. Jensen, and M. B. Nielsen, "Ultrasonic colour Doppler imaging," Interface Focus, vol. 1, no. 4, pp. 490-502, 2011. 